\title{
Classification of Mild Cognitive Impairment by Fusing Neuroimaging and Gene Expression Data
}

\author{
Classification of Mild Cognitive Impairment by Fusing Neuroimaging and Gene Expression Data
}

\author{
Yanjun Lyu \\ Department of Computer Science and \\ Engineering, University of Texas at \\ Arlington, Arlington, Texas, USA \\ yx19168@mavs.uta.edu
}

\author{
Xiaowei Yu \\ Department of Computer Science and \\ Engineering, University of Texas at \\ Arlington, Arlington, TX, USA \\ xxy1302@mavs.uta.edu
}

\author{
Lu Zhang \\ Department of Computer Science and \\ Engineering, University of Texas at \\ Arlington, Arlington, TX, USA \\ lu.zhang2@mavs.uta.edu
}

\author{
Dajiang Zhu \\ Department of Computer Science and \\ Engineering, University of Texas at \\ Arlington, Arlington, TX, USA \\ dajiang.zhu@uta.edu
}

and For the Alzheimer's

Disease Neuroimaging

Initiative ${ }^{*}$

\begin{abstract}
As reversing the pathology of Alzheimer's disease (AD) is impossible, the diagnosis of mild cognitive impairment (MCI), which is considered as the precursor of $\mathrm{AD}$, has become a more tractable goal. Because both brain structural and functional alterations have been observed in $\mathrm{MCI}$ patients, many multimodal fusion approaches have been proposed to classify MCI from normal controls (NC) in clinical studies. Given the complex relationships between brain structure and function, deep learning based models can be helpful in revealing potential non-linear relationships buried in multimodal neuroimaging data. Meanwhile, RNA expression microarray profile can be a complementary feature in brain diseases analysis from another aspect, that is, the knowledge from molecular biology and genetics may benefit the classification of $\mathrm{AD} / \mathrm{MCI}$ patients. To incorporate both imaging and molecular biomarkers, we propose a new deep fusion model: by integrating a cross-model deep network working on multi-modal brain image data and a fully-connected neural network working on gene expression data, a parameter representing the ratio of imaging and genetic features can be learned automatically during the classification process. Using the Alzheimer's Disease Neuroimaging Initiative (ADNI) dataset, this method achieves an overall $82.3 \%$ accuracy, by fusing brain structural and functional connectivity as well as gene expression intensity information.
\end{abstract}

${ }^{*}$ Data used in preparation of this article were obtained from the Alzheimer's Disease Neuroimaging Initiative (ADNI) database (adni.loni.usc.edu)

Permission to make digital or hard copies of all or part of this work for personal or classroom use is granted without fee provided that copies are not made or distributed for profit or commercial advantage and that copies bear this notice and the full citation on the first page. Copyrights for components of this work owned by others than ACM must be honored. Abstracting with credit is permitted. To copy otherwise, or republish, to post on servers or to redistribute to lists, requires prior specific permission and/or a fee. Request permissions from permissions@acm.org.

PETRA 2021, June 29-Fuly 02, 2021, Corfu, Greece

(C) 2021 Association for Computing Machinery.

ACM ISBN 978-1-4503-8792-7/21/06 ..\$15.00

https://doi.org/10.1145/3453892.3453906

\section{CCS CONCEPTS}

- Computing methodologies; • Machine learning; • Machine learning approaches; • Neural networks;

\section{KEYWORDS}

Multi-modality, Brain imaging feature, Gene expression, Deep Model fusion

\section{ACM Reference Format:}

Yanjun Lyu, Xiaowei Yu, Lu Zhang, Dajiang Zhu, and and For the Alzheimer's Disease Neuroimaging Initiative. 2021. Classification of Mild Cognitive Impairment by Fusing Neuroimaging and Gene Expression Data: Classification of Mild Cognitive Impairment by Fusing Neuroimaging and Gene Expression Data. In The 14th PErvasive Technologies Related to Assistive Environments Conference (PETRA 2021), June 29-7uly 02, 2021, Corfu, Greece. ACM, New York, NY, USA, 7 pages. https://doi.org/10.1145/3453892.3453906

\section{INTRODUCTION}

Alzheimer's disease (AD) is the most prevalent form of dementia and is expected to affect 1 out of 85 people in the world by the year 2050 [1]. So far, there is no way to reverse the pathology of Alzheimer's disease $(\mathrm{AD})$ and researchers have been focusing on intervening in its earlier stage - mild cognitive impairment (MCI), which is considered as the precursor of $\mathrm{AD}$. Therefore, establishing a set of objective and non-invasive biomarkers, e.g., imaging markers, is extremely critical. Previous studies reported that the alterations of grey matter (GM) and whiter matter (WM) can be valuable biomarkers of neurodegeneration [2], such as hippocampi volume change [3] and gray matter atrophy [4] observed via structure magnetic resonance imaging (sMRI), white matter disruption [5] using diffusion tensor imaging (DTI), abnormal functional connectivity in default mode network (DMN) [6] using functional MRI, and hypometabolism in the temporal and parietal lobe using FDG-PET [7]. These biomarkers together may yield complementary information, i.e., different modalities can capture disease information from different perspectives, to improve the fundamental understanding of the disease related patterns that cannot be detected using single modality. 
To effectively combine different modalities, numerous multimodal fusion approaches have been developed for cognitive and clinical studies $[8,9]$. One widely used fusion strategy is to extract features from each modality separately, and then perform association analysis, e.g., regression or correlation. Previous studies have demonstrated the complexity between the information conveyed by different types of data $[8,11]$, however, most current fusion methods have been focusing on simple and linear relationship across different modalities [10]. Recently, many studies applied deep learning based models to brain imaging analysis and reported that deep learning strategy can be very efficient to reveal complex and non-linear relationships buried in the raw imaging data. For example, convolutional neural network $(\mathrm{CNN})$ was used to conduct $\mathrm{AD}$ classification based on structural MRI and PET image [12]. Graph Convolutional Network (GCN) was adopted for modeling the MCI conversion [13]. More importantly, we can leverage deep learning methods to explore the potential "deep" associations among imaging markers, genetic markers and the clinical outcomes, which may be difficult to detect using shallow models. For example, RNA profiling data has the potential to be important biomarkers in distinguishing the MCI patients. Previous studies have used blood expression data and traditional machine learning (ML) methods $[14,15]$ to classify MCI patients as well as using multi blood gene expression datasets [16]. The latest study adopted imaging-transcriptomic analysis and suggested that there are some complex associations between transcriptome data and brain connectomes [17].

Inspired by the abovementioned work, in this paper we integrated the brain data collected from multiple domains, including DTI derived structural connectome, functional signals and the gene expression via a carefully designed deep fusion model (Figure. 1). Considering that the RNA profiling data is completely distinct from the other two imaging-based datatypes (structural connectome and functional signals), our deep model is divided into two parts to handle imaging data and genetic data separately. Similar to our previous work, we adopted an RNN/GCN-Attention combined structure to integrate both brain spatial and temporal features (left part in Figure 1). For the expression data, a full connected neural network was employed (right part in Figure 1), expecting to capture the linear interaction among gene loci by their expression intensity value. At last, the output of these two parts (semi-predict) are combined via a trainable parameter, which represents the ratio during the combination, for classification.

\section{METHOD}

\subsection{Overview}

In this work, the gene expression data are involved in supporting the cross-model deep network which is designed to work on multi-modality features extracted from brain image data. Guided by the cross-datatype merging strategy, we proposed a cross-datatype deep fusion model (CDF-model) to combine brain imaging features and genetic features to conduct $\mathrm{MCI} / \mathrm{NC}$ classification. The CDFmodel was composed by two main parts: image component part and gene component part. Forward along the whole fusion-model, the two semi-predicted outputs from the two parts were combined in a learnable way. In section 2, we first introduced the data collection and pre-processing procedures for both imaging and gene expression data (Section 2.2), then the details of image component model (Section 2.3.1), gene component model (Section 2.3.2) and the fusion strategy are followed.

\subsection{Data collection and preprocessing}

In this work, all the data used is obtained from Alzheimer's Disease Neuroimaging Initiative (ADNI) [18]. At the beginning, there are 661 subjects with both DTI and rs-fMRI data available. After the pre-processing and image quality check, 452 subjects were removed. Meanwhile, the number of subjects which have RNA profiling data is 742 . The intersection set of the two sample sets were collected which contain 126 subjects $(63 \mathrm{CN} / 63 \mathrm{MCI})$.

2.2.1 Image data description and preprocessing. DTI data is $2.0 \mathrm{~mm}$ isotropic, $\mathrm{TE}=56 \mathrm{~ms}, \mathrm{TR}=7.2 \mathrm{~s}$, and the gradient direction is 54 . For rsfMRI, the range of image resolution in $\mathrm{X}$ and $\mathrm{Y}$ dimensions is from $2.29 \mathrm{~mm}$ to $3.31 \mathrm{~mm}$. The range of slice thickness is from $3.3 \mathrm{~mm}$ to $3.4 \mathrm{~mm}, \mathrm{TE}=30 \mathrm{~ms}, \mathrm{TR}=3 \mathrm{~s}$, and there are 197 volumes (time points) for each subject. We applied the same standard pre-processing procedures as in [19].

Destrieux [20] atlas been adopted to divide the whole brain into different regions, which can be used as nodes. The structural connectivity matrices $A \in R^{N \times N}, N$ is the number of regions, have been created for each subject. The weights are computed by counting the number of fibers connecting region pairs. By discarding the regions with all zero values, $\mathrm{N}=148$ is the number of remaining brain regions. For each brain region, the averaged fMRI BOLD signals have been used as the temporal features $F \in R^{N \times T}, T$ is the number of time points. In addition, a data augmentation step was introduced to acquire more training samples. Specifically, the original signal from each subject was divided into 4 segments (each covered 45 time points). Previous study [21] indicates that 20 time points (40s) incline to be sufficient to capture major functional dynamics in resting state fMRI. To maintain the completeness of data on each subject, we simply copied the corresponding structural connectivity data and gene expression data for the augmented samples.

2.2.2 Blood gene expression dataset. The Blood RNA expression microarray profiling data was obtained from ADNI LONI Web site (http://adni.loni.usc.edu). The ADNI study have collected 811 whole blood samples which were processed using Qiagen PAXgene Blood RNA Kit (Germantwon, MD, USA) [22]. The expression data was generated by array hybridization on the Affymetrix Human Genome U219 array plate (Affymetrix, Santa Clara, CA, USA), and preprocessed with robust normalization and quality-controlled [23]. Two further steps were taken to reduce the background noise in the gene expression data and dump the probes to certain gene. First, the probe-sets with maximal expression intensity value lower than the median among all probe-sets intensity were removed. Then, if serval probes were mapped to the same gene, the one with the highest variance among the samples was selected to represent the particular gene, followed the procedures in [24]. After these two steps, 16754 genes with expression level values were selected and been put to Lasso feature selection. 


\section{Brain Imaging Modality}

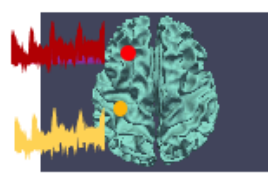

fMRI Signal
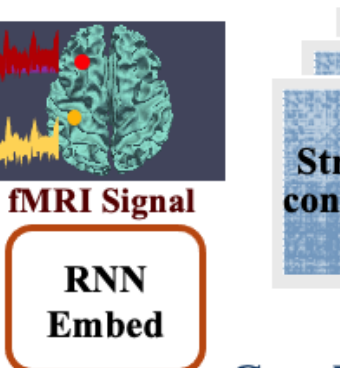

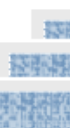

Structural connectivity

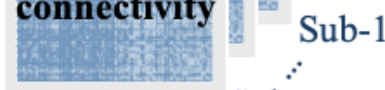

Sub-n

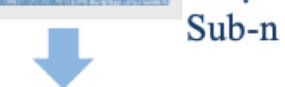

Graph Model

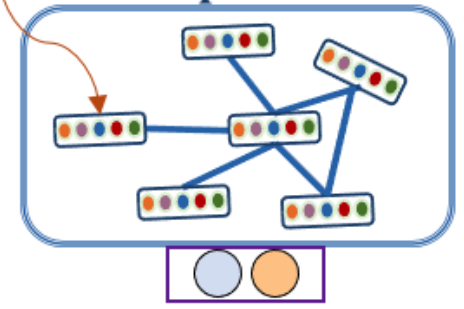

Imaging Semi-Predict
Blood Gene Expression

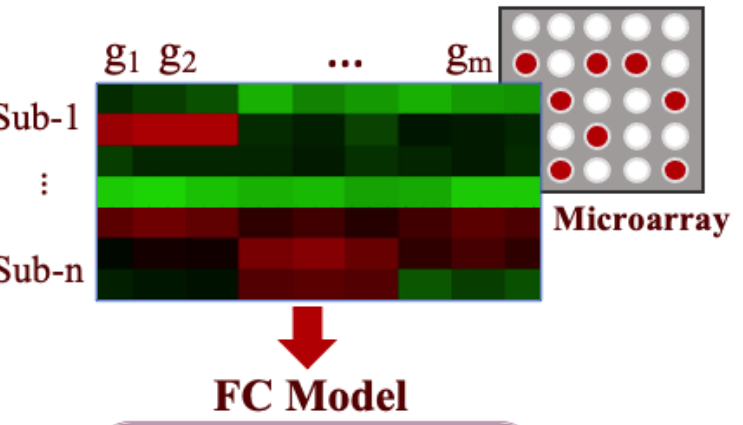

\section{Classifier (NC vs MCI)}

Figure 1: Illustration of our cross-datatype fusion model. An RNN/GCN-Attention combined deep model is designed to fuse the multi-modal brain imaging data (left). A fully connected neural network is designed to model gene expression data (right). Two models are merged in the final step via a trainable parameter.

\subsection{Deep fusion model with cross-datatype support}

In this work, a cross-datatype deep fusion model (CDF-Model) is designed to classify MCI patients from normal controls (NC). The model is composed of two parts. The first part is a cross-model attention network, which takes the multi-modality image data as input, one RNN layer and multiple graph attention (GAT) blocks were applied. Another part is single one-layer fully connected network which takes gene expression features as input. Both the outputs of the two parts could be treated as semi-classifying prediction towards the MCI and NC, by learnable weights, and the two semiprediction are combined to make a final prediction.

2.3.1 Image component. Following the previous work on using deep model to address multi-modality brain image data, the image component model consists of one LSTM layer and several GCNAttention (GAT) blocks [25].

The LSTM layer is responsible for capturing the dynamic temporal features of fMRI signals at certain brain regions while the GAT blocks capture both the functional correlations between different brain regions and the connectivity features for each region from its neighbors.

The LSTM [26, 27] extends the basic RNN [28] by adding three gates: forget gate $f$, input gate $i$ and output gate $o$, which allow LSTM to capture long-term dependency and make it easier to optimize [25]. In LSTM formulas, $\sigma$ is the activation function, matrices $\left\{\boldsymbol{W}_{f}\right.$, $\left.\boldsymbol{W}_{i}, \boldsymbol{W}_{c}, \boldsymbol{W}_{o}\right\}$ and vectors $\left\{\boldsymbol{b}_{f}, \boldsymbol{b}_{i}, \boldsymbol{b}_{c}, \boldsymbol{b}_{o}\right\}$ are parameters which need to be learned. $x_{t}$ is the input feature vector at $t^{\text {th }}$ timestamp and $\boldsymbol{h}_{\boldsymbol{t}-1}$ is the output of previous LSTM at the $t-1^{\text {th }}$ timestamp. The $f, i$ and $o$ denote the forget gate, input gate and output gate respectively.

$$
\begin{gathered}
\mathrm{f}_{\mathrm{t}}=\sigma\left(\boldsymbol{W}_{\mathrm{f}} \cdot\left[\boldsymbol{h}_{\mathrm{t}-1}, \boldsymbol{x}_{\mathrm{t}}\right]+\boldsymbol{b}_{f}\right) \\
\mathrm{i}_{\mathrm{t}}=\sigma\left(\boldsymbol{W}_{\mathrm{i}} \cdot\left[\boldsymbol{h}_{\mathrm{t}-1}, \boldsymbol{x}_{\mathrm{t}}\right]+\boldsymbol{b}_{i}\right) \\
\mathrm{C}_{\mathrm{t}}^{\prime}=\tanh \left(\boldsymbol{W}_{\mathrm{c}} \cdot\left[\boldsymbol{h}_{\mathrm{t}-1}, \boldsymbol{x}_{t}\right]+\boldsymbol{b}_{\boldsymbol{c}}\right) \\
\mathrm{C}_{\mathrm{t}}=\mathrm{f}_{\mathrm{t}} * \mathrm{C}_{\mathrm{t}-1}+\mathrm{i}_{\mathrm{t}} * \mathrm{C}_{\mathrm{t}}^{\prime} \\
\boldsymbol{o}_{t}=\sigma\left(\boldsymbol{W}_{\mathrm{o}} \cdot\left[\boldsymbol{h}_{\mathrm{t}-1}, \boldsymbol{x}_{\mathrm{t}}\right]+\boldsymbol{b}_{o}\right)
\end{gathered}
$$




$$
\boldsymbol{h}_{\mathrm{t}}=\boldsymbol{o}_{\mathrm{t}} * \tanh \left(\mathrm{C}_{\mathrm{t}}\right)
$$

In GAT blocks, the Attention layers take the dynamic temporal functional features captured by the previous RNN layer as input. The attention operations were made region-wise. The proposed attention mechanism is implemented by a single-layer feedforward neural network and parameterized by weight vector $\alpha^{T} . N$ is the number of brain regions, and $F$ is the number of functional features, the input of attention layer is $\boldsymbol{h}=\left\{\boldsymbol{h}_{1}, \boldsymbol{h}_{2}, \ldots, \boldsymbol{h}_{\boldsymbol{N}}\right\}, \boldsymbol{h}_{\boldsymbol{i}} \in \boldsymbol{R}^{F}$. Given a shared linear transformation weight matrix $W \in R^{F^{\prime} \times F}$, attention mechanism is implemented by a single-layer feedforward neural network and parameterized by weight vector $\alpha^{T} \in R^{2 F^{\prime}}$. Attention mechanism is calculated by:

$$
\alpha_{i j}=\operatorname{Re} L U\left(\alpha^{T}\left[W h_{i} \| W h_{j}\right]\right)
$$

Here, the $\alpha_{\mathrm{ij}}$ indicates the importance of the feature of region $\mathrm{j}$ to the feature of region $i$, and operation $\|$ means the concatenation. Then the feature on region $i$ could be reorganized by:

$$
\boldsymbol{h}_{i}^{\prime}=\operatorname{ReLU}\left(\sum_{j} \alpha_{i j} \boldsymbol{W} \boldsymbol{h}_{j}\right)
$$

Subsequently, two GCN layers were stacked to integrate the features from distant neighbors (second order neighbors) for each vertex on the structural connectome graph.

$$
G(\boldsymbol{A}, \boldsymbol{F})=\operatorname{ReLU}\left(\mathrm{A} \operatorname{ReLU}\left(\boldsymbol{A} \boldsymbol{F} \boldsymbol{W}_{0}\right) \boldsymbol{W}_{1}\right)
$$

$\boldsymbol{W}$ is the weight matrix of each layer, $\boldsymbol{A}$ is the adjacency matrix and $\boldsymbol{F}$ is the feature matrix. The outputs of the second GCN layer are fed into a fully-connected layer for producing a semi-prediction of classification

2.3.2 Gene component. The gene component model is set to a simple one-hidden layer neural network. In order to output a semiclassifying prediction, the last layer of this sub-model have two nodes. It is noteworthy that there is no nonlinear active function here since the aim of this sub-model is to capture the linear interactive relationship across different genes.

$$
s=W_{2} \cdot\left(W_{1} \cdot g+b_{1}\right)+b_{2}
$$

The $s$ is final output (semi-prediction) of the sub-model, $g$ indicates the input pre-normalized gene expression features. The coefficients $\left\{\boldsymbol{W}_{1}, \boldsymbol{W}_{2}\right\},\left\{\boldsymbol{b}_{1}, \boldsymbol{b}_{2}\right\}$ are weights and bias for the hidden layers and the output layer.

2.3.3 Fusion of semi-predictions. At last, the outputs (semiprediction) of both parts are feed into a fusion layer. For the image component sub-model, the $\tilde{P}_{L, 0}$ and $\tilde{\mathrm{P}}_{\mathrm{L}, 1}$ stand for the semiprediction of label ' 0 ' or ' 1 ', analogously, the $\tilde{P}_{R, 0}$ and $\tilde{P}_{R, 1}$ stand for the output of the gene component sub-model. The $\left[\begin{array}{l}\theta_{L} \\ \theta_{R}\end{array}\right]$ is the learnable coefficient to assign weights to the semi-prediction of both parts and $\left[\begin{array}{l}P_{0} \\ P_{1}\end{array}\right]$ is the final fusion prediction. $\gamma$ is the adjusting coefficient added to ensure the outputs of two sub-model are within the same scale.

$$
\left[\begin{array}{l}
P_{0} \\
P_{1}
\end{array}\right]=\left[\begin{array}{ll}
\tilde{P}_{L, 0} & \gamma \tilde{P}_{R, 0} \\
\tilde{P}_{L, 1} & \gamma \tilde{P}_{R, 1}
\end{array}\right] \cdot \operatorname{Softmax}\left(\left[\begin{array}{l}
\theta_{L} \\
\theta_{R}
\end{array}\right]\right)
$$

\section{RESULTS}

The binary classification task (NC vs MCI) was performed on our CDF-Model. The model takes the multi-modality image data and gene expression data as input. In order to alleviate the possible overfitting brought by the small sample size and test the reproducibility of the results, a cross validation experiment was applied. In the results part, the classification accuracy is showed at first (Section 3.2), followed by showing potential significant brain regions detected in the model (Section 3.3) and the model evaluation (Section 3.4).

\subsection{Model setting}

Given the dataset with $63 \mathrm{CN}$ samples and $63 \mathrm{MCI}$ samples, we adopted four-folds cross-validation to report our results. For the structure of image component model, temporal features of fMRI singles from different brain regions are first extracted by a RNN layer (LSTM with hidden size 128), then two graph convolution attention layer blocks (Output dimension: 128 and 256) were applied to transform the signal features on structural connectivity graph. The hyper-parameters of model were determined based on previous works reported in [20], which are then adjusted to fit our sample size. This model was pre-trained on a separate imaging dataset (241 $\mathrm{CN}, 108 \mathrm{MCI}$ ) from ADNI, and the trained model with test accuracy of $81.9 \%$ has been taken to initialize the image model in the fusion process.

The Gene component model is a two-layers (size 128 and 2) fullyconnected neural network. In order to reduce the dimensionality of the gene expression data (16754 features), a lasso feature selection (with alpha $=0.000001$ ) process was employed and the remaining size of the gene expression features is 1040 .

During the training process, a 'StepLR' scheduler was applied to adjust the learning rate during the training. For each fold in cross-validation, the best test accuracy was recorded as the test accuracy.

\subsection{Classification performance}

3.2.1 Performance of pretrained single model and fusion model. According to Table 1, the overall accuracy of our CDF-Model is $82.3 \%$ on the cross-validation dataset and $79.8 \%$ on $1: 1$ trainingtesting dataset. Whereas the two single models, image component model and gene component model, reached accuracies of $78.5 \%$ and $79.0 \%$ respectively on the 1:1 training-testing dataset.

All the accuracy reported below is the average of 5 runs. Note that we reported our CDF results of both four-folds cross-validation and 1:1 split in Table 1. For pretrained models including image and genetic component we only include $1: 1$ split results. Precision, recall and F1 score are based on the average of the best records and are also reported here.

3.2.2 Performance comparison in $\mathrm{CN} / \mathrm{MCl}$ classification. We summarized some other recent studies for $\mathrm{CN} / \mathrm{MCI}$ classification and the results are listed in Table 2. For fair comparison, all the literatures listed here are designed for multi-modality data classification.

\subsection{Top MCI-related brain regions}

One advantages of GCN-Attention blocks used in this model is that the importance of features corresponding to each node could 
Table 1: Classification performance

\begin{tabular}{lllll}
\hline Model & Test Accuracy(avg) & Precision & Recall & F1 Score \\
\hline CDF-Model (Cross Validation) & $\mathbf{8 2 . 3 \%}$ & $\mathbf{8 3 . 9 6 \%}$ & $\mathbf{8 4 . 6 5 \%}$ & $\mathbf{8 4 . 1 7 \%}$ \\
CDF-Model & $\mathbf{7 9 . 8 \%}$ & $\mathbf{8 1 . 1 5 \%}$ & $\mathbf{8 0 . 8 8 \%}$ & $\mathbf{8 0 . 9 1 \%}$ \\
Image Component Model & $78.5 \%$ & $79.00 \%$ & $82.41 \%$ & $80.55 \%$ \\
Gene Component Model & $79.0 \%$ & $79.34 \%$ & $82.12 \%$ & $80.67 \%$ \\
\hline
\end{tabular}

Table 2: Performance of different methods in CN/MCI classification (Multi-Modality Studies)

\begin{tabular}{|c|c|c|c|}
\hline Study & Sample size & Methods & Acc \\
\hline Yu et al, 2016 [29] & $52 \mathrm{CN}, 97 \mathrm{MCI}$ & $\begin{array}{l}\text { ROI-based; Graph-guided } \\
\text { learning }\end{array}$ & MCI vs $\mathrm{CN} 80.0 \%$ \\
\hline Tong et al, 2017 [30] & $35 \mathrm{CN}, 75 \mathrm{MCI}$ & Nonlinear Graph-fusion & MCI vs CN $79.5 \%$ \\
\hline Suk et al, 2017 [31] & $\begin{array}{l}226 \mathrm{CN}, 167 \text { converters } \mathrm{MCI} \text {, } \\
226 \text { stable } \mathrm{MCI}\end{array}$ & Multiple sparse & MCI vs CN $66.78 \%-73.02 \%$ \\
\hline Aderghal et al, 2018 [32] & $58 \mathrm{CN}, 108 \mathrm{MCI}$ & $\begin{array}{l}\text { Hippocampus; CNN; Transfer } \\
\text { learning }\end{array}$ & $\mathrm{MCI}$ vs $\mathrm{CN} 80 \%$ \\
\hline CDF-Model (This work) & $63 \mathrm{CN}, 63 \mathrm{MCI}$ & Fusion model & MCI vs CN $82.3 \%$ \\
\hline
\end{tabular}

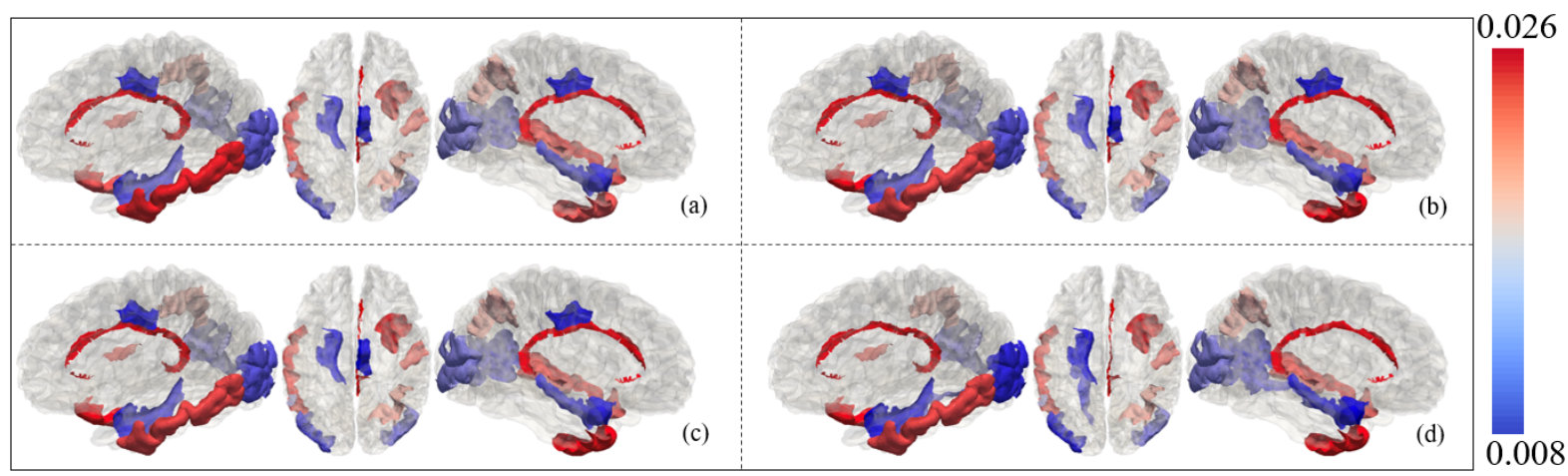

Figure 2: Brain map of significant MCI-related brain regions. The color indicates the significant value corresponding of the region. The higher the value is, the more likely that the features of this region are related to MCI-CN classification. Across all four-fold cross validation experiments (a-d), the high related regions are constant.

be recovered. By analyzing the image component model part in the trained CDF-Model, the weights of the bottleneck layer can be matched back to each one of the 148 regions using Laplacian Score (LSCORE) feature extraction method. The summations of such weights give the importance of the regions.

As showing in Figure 2, the highlighted top $10 \mathrm{MCI}-\mathrm{CN}$ classification related brain regions are highly consistent across each of the four-fold cross validation experiments. In total, 11 regions were detected, and the scale of significant value is from 0.008 to 0.026 . The significant values are the summation of the corresponding weights of bottleneck layer in the optimized model. We also showed the names of the 11 regions and their significant values in Table 3

\subsection{Model evaluation}

The CDF-Model was tested on a 4-fold cross-validation experiment. Since the pre-train process has been implemented on the image component sub-model, in each fold the model converged fast (Figure 3). Due to the small sample size, different partitions of the samples (for training and testing) have influence on model convergence in different folds.

\section{CONCLUSION}

In this work, we developed a deep cross-model cross-datatype network to integrate brain imaging data and gene expression data for jointly classifying MCI patients. In order to simultaneously capture brain temporal dynamics, structural connectome and their associations, an RNN/GCN-Attention model is used to manipulate multimodal neuroimaging data. By introducing the information from gene expression profile, the classification performance is slightly improved. Our results demonstrate that genetic data may contain complementary information to imaging data and that knowledge can be helpful in early diagnosis of MCI. 
Table 3: The 11 MCI-related regions

\begin{tabular}{llll}
\hline Location & Significant Value (Avg.) & Location & $\begin{array}{l}\text { Significant Value } \\
\text { (Avg.) }\end{array}$ \\
\hline lh_G_temporal_middle_lager & 0.0184 & rh_S_pericallosal_lager & 0.0256 \\
rh_Pole_temporal_lager & 0.0180 & lh_G_occipital_middle_lager & 0.0156 \\
rh_G_temp_sup-G_T_transv_lager & 0.0175 & rh_G_occipital_middle_lager & 0.0160 \\
rh_S_intrapariet\&P_trans_lager & 0.0174 & lh_G_oc-temp_med-Paraphip_lager & 0.0089 \\
lh_S_interm_prim-Jensen_lager & 0.0171 & lh_G_oc-temp_med\&Lingual_lager & 0.0158 \\
lh_G\&S_cingul-Mid-Post_lager & 0.0089 & & \\
\hline
\end{tabular}
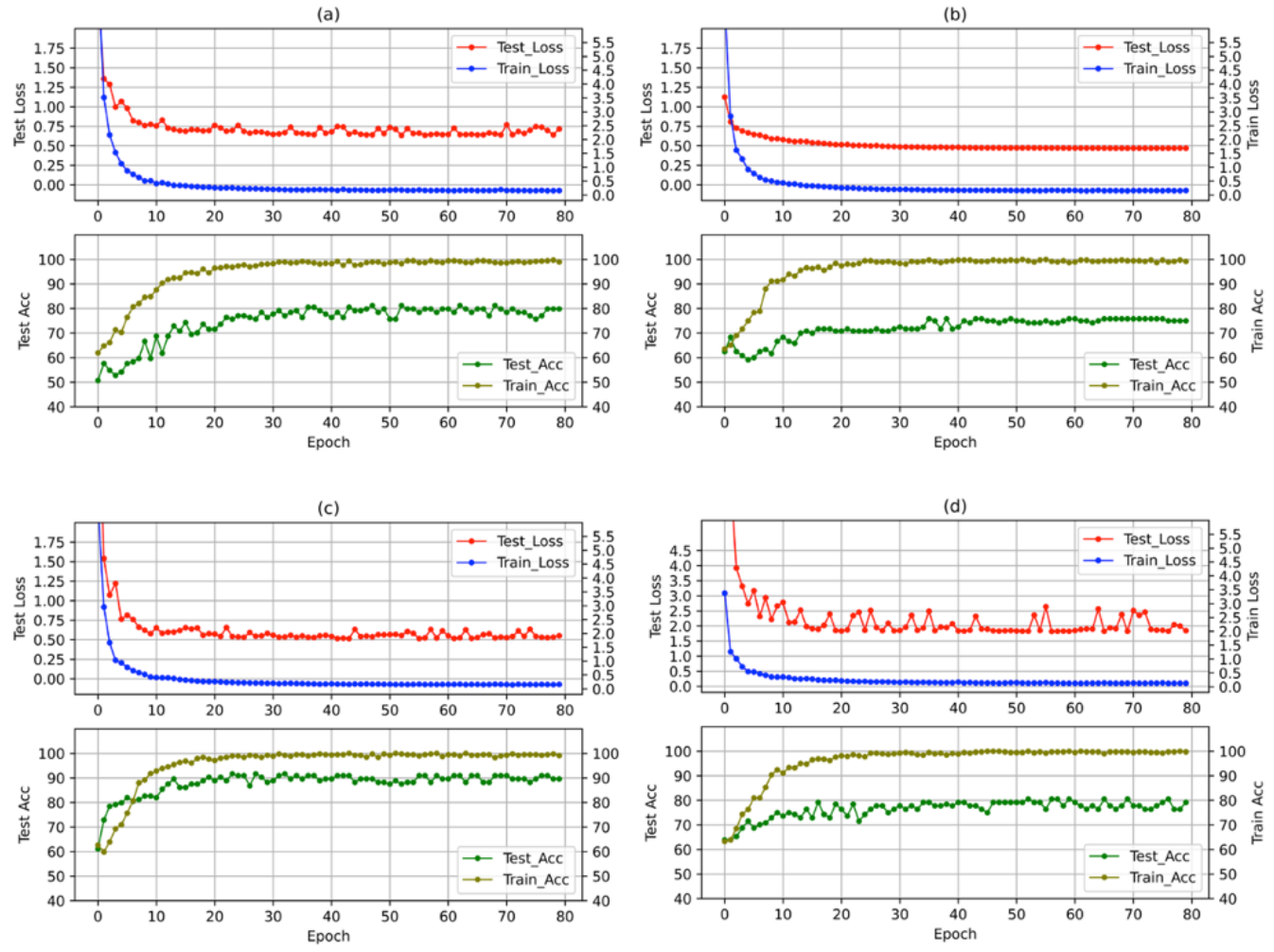

Figure 3: The results of four-fold cross validation (a-d). In each sub graph, the upper graph stands for convergence curve of training loss and testing loss, the lower graph stands for convergence curve of training accuracy and testing accuracy.

\section{REFERENCES}

[1] Brookmeyer R, Johnson E, Ziegler-Graham K, Arrighi HM. 2007. Forecasting the global burden of Alzheimer's disease. Alzheimers Dement. 2007 Jul;3(3):186-91. DOI: https://doi.org/10.1016/j.jalz.2007.04.381

[2] Frisoni, G. B., Fox, N. C., Jack, C. R., Jr, Scheltens, P., \& Thompson, P. M. 2010. The clinical use of structural MRI in Alzheimer disease. Nature reviews. Neurology, 6(2), 67-77. DOI: https://doi.org/10.1038/nrneurol.2009.215

[3] Henneman, W. J., Sluimer, J. D., Barnes, J., van der Flier, W. M., Sluimer, I. C., Fox, N. C., Scheltens, P., Vrenken, H., \& Barkhof, F. 2009. Hippocampal atrophy rates in Alzheimer disease: added value over whole brain volume measures. Neurology, 72(11), 999-1007. DOI: https://doi.org/10.1212/01.wnl.0000344568.09360.31

[4] Karas, G. B., Scheltens, P., Rombouts, S. A., Visser, P. J., van Schijndel, R. A., Fox, N. C., \& Barkhof, F. 2004. Global and local gray matter loss in mild cognitive impairment and Alzheimer's disease. NeuroImage, 23(2), 708-716. DOI: https: //doi.org/10.1016/j.neuroimage.2004.07.006

[5] Li, S., Pu, F., Shi, F., Xie, S., Wang, Y., \& Jiang, T. 2008. Regional white matter decreases in Alzheimer's disease using optimized voxel-based morphometry. Acta radiologica (Stockholm, Sweden: 1987), 49(1), 84-90. DOI: https://doi.org/10.
1080/02841850701627181

[6] Mevel, K., Chételat, G., Eustache, F., \& Desgranges, B. 2011. The default mode network in healthy aging and Alzheimer's disease. International journal of Alzheimer's disease, 2011, 535816. DOI: https://doi.org/10.4061/2011/535816

[7] Langbaum, J.B., Chen, K., Lee, W., Reschke, C., Bandy, D., Fleisher, A.S., Alexander, G.E., Foster, N.L., Weiner, M.W., Koeppe, R.A., et al. 2009. Categorical and correlational analyses of baseline fluorodeoxyglucose positron emission tomography images from the Alzheimer's Disease Neuroimaging Initiative (ADNI). Neuroimage 45, 1107-1116. DOI: https://doi.org/10.1016/j.neuroimage.2008.12.072

[8] Honey, C. J., Sporns, O., Cammoun, L., Gigandet, X., Thiran, J.-P., Meuli, R., \& Hagmann, P. 2009. Predicting human resting-state functional connectivity from structural connectivity. Proceedings of the National Academy of Sciences, 106, 2035-2040. DOI: https://doi.org/10.1073/pnas.0811168106

[9] Huang, Y., Xu, J., Zhou, Y., Tong, T., Zhuang, X., \& Alzheimer's Disease Neuroimaging Initiative (ADNI). 2019. Diagnosis of Alzheimer's Disease via MultiModality 3D Convolutional Neural Network. Frontiers in neuroscience, 13, 509. DOI: https://doi.org/10.3389/fnins.2019.00509.

[10] Plis, S. M., Amin, M. F., Chekroud, A., Hjelm, D., Damaraju, E., Lee, H. J., Bustillo, J. R., Cho, K., Pearlson, G. D., \& Calhoun, V. D. 2018. Reading the (functional) 
writing on the (structural) wall: multimodal fusion of brain structure and function via a deep neural network based translation approach reveals novel impairments in schizophrenia. Neuroimage, 181, 734-747.

[11] Li, K., Guo, L., Zhu, D. et al. 2012. Individual Functional ROI Optimization Via Maximization of Group-Wise Consistency of Structural and Functional Profiles. Neuroinform 10, 225-242 (2012). DOI: https://doi.org/10.1007/s12021-012-9142-5

[12] Liu M, Zhang J, Adeli E, Shen D. 2017. Deep multi-task multi-channel learning for joint classification and regression of brain status. In International conference on medical image computing and computer-assisted intervention, Springer, Cham, pp. (3-11).

[13] Liu M, Zhang J, Adeli E, Shen D. 2017. Deep Multi-Task Multi-Channel Learning for Joint Classification and Regression of Brain Status. Med Image Comput Comput Assist Interv. 2017 Sep; 10435:3-11. DOI: https://doi.org/10.1007/978-3319-66179-7_1

[14] Li, H., Hong, G., Lin, M. et al. 2017. Identification of molecular alterations in leukocytes from gene expression profiles of peripheral whole blood of Alzheimer's disease. Sci Rep 7, 14027. DOI: https://doi.org/10.1038/s41598-017-13700-w

[15] Li, X., Wang, H., Long, J. et al. 2018. Systematic Analysis and Biomarker Study for Alzheimer's Disease. Sci Rep 8, 17394. DOI: https://doi.org/10.1038/s41598018-35789-3

[16] Lee, T., Lee, H. 2020. Prediction of Alzheimer's disease using blood gene expression data. Sci Rep 10, 3485. DOI: https://doi.org/10.1038/s41598-020-60595-1

[17] Woo, Y.J., Roussos, P., Haroutunian, V. et al. 2020. Comparison of brain connec tomes by MRI and genomics and its implication in Alzheimer's disease. BMC Med 18, 23. DOI: https://doi.org/10.1186/s12916-019-1488-1

[18] ADNI | Alzheimer's Disease Neuroimaging Initiative, http://adni.loni.usc.edu/

[19] Lu Zhang, et al. 2019. A Cascaded Multi-Modality Analysis in Mild Cognitive Im pairment. 10th International Workshop on Machine learning in Medical Imaging (MLMI 2019), held in Conjunction with MICCAI 2019, in pressing.

[20] Destrieux, C., Fischl, B., Dale, A., \& Halgren, E. 2010. Automatic parcellation of human cortical gyri and sulci using standard anatomical nomenclature. NeuroImage, 53(1), 1-15. DOI: https://doi.org/10.1016/j.neuroimage.2010.06.010

[21] Zhang, X., Guo, L., Li, X., Zhang, T., Zhu, D., Li, K., Chen, H., Lv, J., Jin, C., Zhao, Q., Li, L., \& Liu, T. 2013. Characterization of task-free and task-performance brain states via functional connectome patterns. Medical image analysis, 17(8) 1106-1122. DOI: https://doi.org/10.1016/j.media.2013.07.003

[22] Saykin, A. J., Shen, L., Yao, X., Kim, S., Nho, K., Risacher, S. L., Ramanan, V. K., Foroud, T. M., Faber, K. M., Sarwar, N., Munsie, L. M., Hu, X., Soares, H. D., Potkin, S. G., Thompson, P. M., Kauwe, J. S., Kaddurah-Daouk, R., Green, R. C.,
Toga, A. W., Weiner, M. W., ... Alzheimer's Disease Neuroimaging Initiative. 2015. Genetic studies of quantitative MCI and AD phenotypes in ADNI: Progress, opportunities, and plans. Alzheimer's \& dementia: the journal of the Alzheimer's Association, 11(7), 792-814. DOI: https://doi.org/10.1016/j.jalz.2015.05.009

[23] Irizarry, R. A., Hobbs, B., Collin, F., Beazer-Barclay, Y. D., Antonellis, K. J., Scherf, U., \& Speed, T. P. 2003. Exploration, normalization, and summaries of high density oligonucleotide array probe level data. Biostatistics (Oxford, England), 4(2), 249264. DOI: https://doi.org/10.1093/biostatistics/4.2.249

[24] Mancarci, B. O., Toker, L., Tripathy, S. J., Li, B., Rocco, B., Sibille, E., \& Pavlidis, P. 2017. Cross-Laboratory Analysis of Brain Cell Type Transcriptomes with Applications to Interpretation of Bulk Tissue Data. eNeuro, 4(6), ENEURO.021217.2017. DOI: https://doi.org/10.1523/ENEURO.0212-17.2017

[25] L. Zhang, L. Wang and D. Zhu. 2020. Jointly Analyzing Alzheimer's Disease Related Structure-Function Using Deep Cross-Model Attention Network. 2020 IEEE 17th International Symposium on Biomedical Imaging (ISBI), Iowa City, IA, USA, 2020, pp. 563-567. DOI: https://doi.org/10.1109/ISBI45749.2020.9098638

[26] Sepp Hochreiter and Jürgen Schmidhuber. 1997. Long Short-Term Memory. Neural Comput. 9, 8 (November 15, 1997), 1735-1780. DOI: https://doi.org/10.1162/ neco.1997.9.8.1735

[27] Ilya Sutskever, Oriol Vinyals, and Quoc V. Le. 2014. Sequence to sequence learning with neural networks. In Proceedings of the 27th International Conference on Neural Information Processing Systems - Volume 2 (NIPS'14). MIT Press, Cambridge, MA, USA, 3104-3112.

[28] Cho, K., Merrienboer, B.V., Gülçehre, Ç., Bahdanau, D., Bougares, F., Schwenk, H., \& Bengio, Y. 2014. Learning Phrase Representations using RNN Encoder-Decoder for Statistical Machine Translation. ArXiv, abs/1406.1078

[29] Yu, G., Liu, Y., \& Shen, D. 2016. Graph-guided joint prediction of class label and clinical scores for the alzheimer's disease. Brain Structure and Function, 221, $3787-3801$

[30] Tong, T., Gray, K., Gao, O., Chen, L., Rueckert, D., Initiative, A. D. N. et al. 2017. Multi-modal classification of alzheimer's disease using nonlinear graph fusion. Pattern recognition, 63, 171- 181.

[31] Suk, H. I., Lee, S. W., Shen, D., \& Alzheimer's Disease Neuroimaging Initiative. 2017. Deep ensemble learning of sparse regression models for brain disease diagnosis. Medical image analysis, 37, 101-113. DOI: https://doi.org/10.1016/j. media.2017.01.008

[32] Aderghal, K., Khvostikov, A., Krylov, A., Benois-Pineau, J., Afdel, K., \& Catheline, G. 2018. Classification of alzheimer disease on imaging modalities with deep cnns using cross-modal transfer learning. In 2018 IEEE 31st International Symposium on Computer-Based Medical Systems (CBMS) (pp. 345-350). IEEE. 